\title{
Primary myxoid liposarcoma of the orbit
}

\author{
CAROL M LANE', J E WRIGHT, ' AND A GARNER \\ From the 'Orbital Clinic, Moorfields Eye Hospital, London EC1V 2PD, and the ${ }^{2}$ Department of Pathology, \\ Institute of Ophthalmology, University of London
}

SUMMARY Orbital liposarcoma is a rare and usually unsuspected neoplasm. Over a five-year period three female patients aged 22,71 , and 77 years presented with primary myxoid liposarcoma of the orbit. The management of one patient was complicated by a history of orbital decompression for suspected thyroid eye disease. The tumour infiltrates locally beyond a deceptive pseudocapsule, and surgery has to be radical to be effective.

The orbit is a rare site for primary liposarcoma, ${ }^{1}$ a tumour which usually originates in deep connective tissue planes and is most common in the thigh and retroperitoneum. Only 10 orbital cases have been reported in detail so far. Four were fatal and six survived to the date of reporting.

Three patients with differing clinical features of myxoid orbital liposarcoma presented to the Orbital Clinic at Moorfields Eye Hospital during the last five years. Their cases illustrate the difficulties which may be experienced in establishing both the diagnosis and the optimum treatment of this tumour. The rationale for surgical excision is discussed in relation to the histological type and the results of studies of systemic liposarcoma.

\section{Patients and methods}

CASE 1

Two years prior to presentation at this clinic a 22 year-old woman developed a painful right proptosis of fairly rapid onset. Myxoedema was diagnosed at the time, and she was treated with thyroxine and systemic steroid for presumed thyroid eye disease. There was no further deterioration during treatment. Five months later the affected orbit was surgically decompressed into the ethmoid and maxillary sinuses, and after temporary improvement the right eye again became progressively proptosed.

When she was first seen in this clinic the visual acuities were $6 / 6$ in both eyes. The right eye was 15 $\mathrm{mm}$ proptosed and displaced $10 \mathrm{~mm}$ downwards and $4 \mathrm{~mm}$ laterally. A firm mass could be palpated in the superomedial quadrant of the right orbit. The cranial

Correspondence to J E Wright, FRCS. nerves were intact apart from the finding of numbness in the medial part of the right upper eyelid. There was no lymphadenopathy.

High resolution computerised tomography confirmed orbital decompression and showed a large heterogeneous mass with areas of negative attenuation (Fig. 1) in the right orbit. A trans-septal orbital biopsy was performed under local anaesthesia.

Histological examination showed inflammatory tissue only. A second biopsy was taken through a

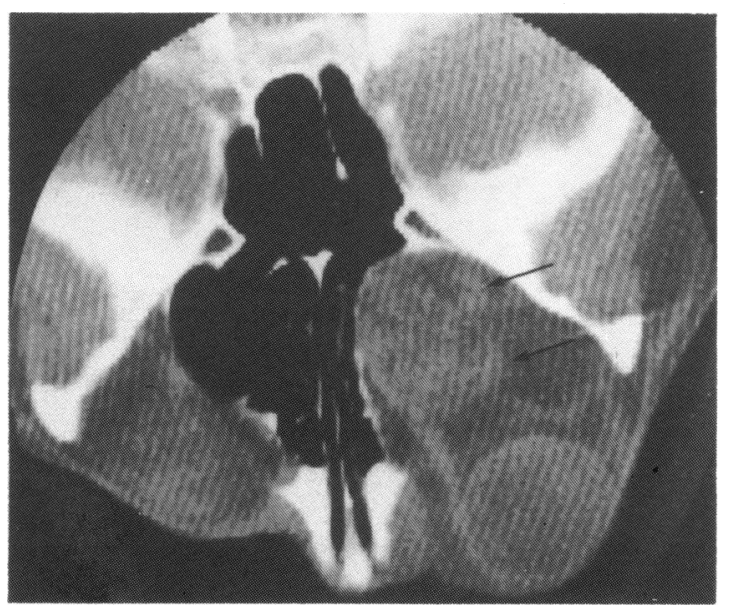

Fig. 1 Axial CT scan in case 1 shows the following features: marked indentation of the ethmoid labyrinth on the affected side due to previous surgical decompression with prolapse of the tumour (arrows) into the decompression; areas of negative attenuation within the tumour mass indicative of the lipomatous nature of the tumour; a generalised increase in total orbital fat content on the affected side. 


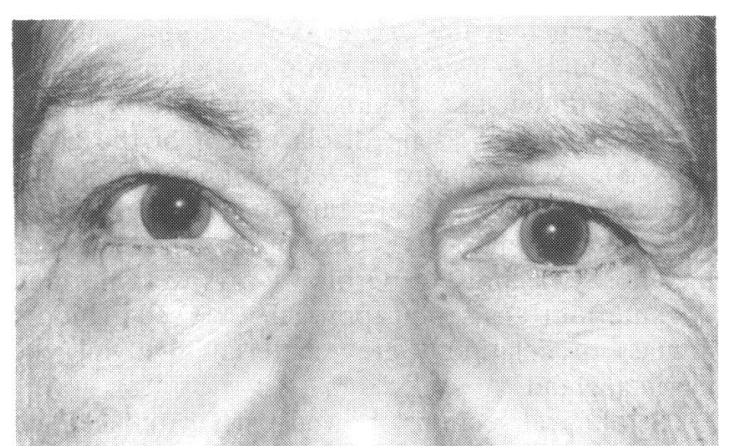

Fig. 2 Case 2 at presentation, with a mass visible beneath the right lower lid.

superomedial conjunctival incision under a general anaesthetic. The diffuse gelatinous tumour appeared to be adherent to and wrapped round the optic nerve and medial rectus muscle.

Initially the histological appearance of a loose, myxoid stroma infiltrated by some spindle shaped cells, eosinophils and mast cells, was suggestive of pseudotumour. However, the stroma contained some alcianophilic acid mucopolysaccharide, and electron microscopy showed many of the cells to contain lipid droplets. After the histological specimens had been referred to a number of centres a diagnosis of myxoid liposarcoma was made.

A short course of steroid therapy was initially given on the basis of the initial diagnosis. Following review of the histology radiotherapy in the form of 20.8 Gray of cobalt-60 treatment was administered. This was followed by craniofacial resection, which included the orbit and maxillary and ethmoidal sinuses. The patient remained alive and well two years later.
CASE 2

A 71-year-old woman noted increasing painless swelling of her right lower eyelid with occasional vertical diplopia over a period of 18 months. Her general health was excellent.

The visual acuities were $6 / 9$ in both eyes with full fields to confrontation. A smooth, bluish swelling was seen below the right lower lid (Fig. 2), and a rubbery, non-tender mass measuring 15 by $30 \mathrm{~mm}$ could be palpated on the right orbital floor. There was $3 \mathrm{~mm}$ of right proptosis and $2 \mathrm{~mm}$ of upward displacement of the right globe, with dilated conjunctival vessels. The ocular movements were full, and there was no relative afferent pupillary defect. Both optic discs were normal. Radiographs showed no abnormality, but computerised tomography revealed an indistinct opaque mass on the floor of the right orbit. Anterior orbitotomy through the lower eyelid revealed an enormous gelatinous mass extending to the orbital apex and attached to the inferior oblique muscle and optic nerve. Subtotal excision was performed.

Histopathology showed a myxoid liposarcoma with delicate vascular channels, characteristic lipoblasts, very few mitoses, and some giant and pleomorphic cells (Fig. 3). Since the lesion was unlikely to be radiosensitive, further resection was considered necessary. However, on readmission for assessment three months later her symptoms had resolved. Retraction of the right upper eyelid had developed and was thought to be related to tethering of the inferior rectus. Computerised tomography showed no evidence of recurrence. Exenteration of the right orbit was suggested to the patient as the only means of ensuring complete removal of the tumour. This treatment was declined but the patient remained

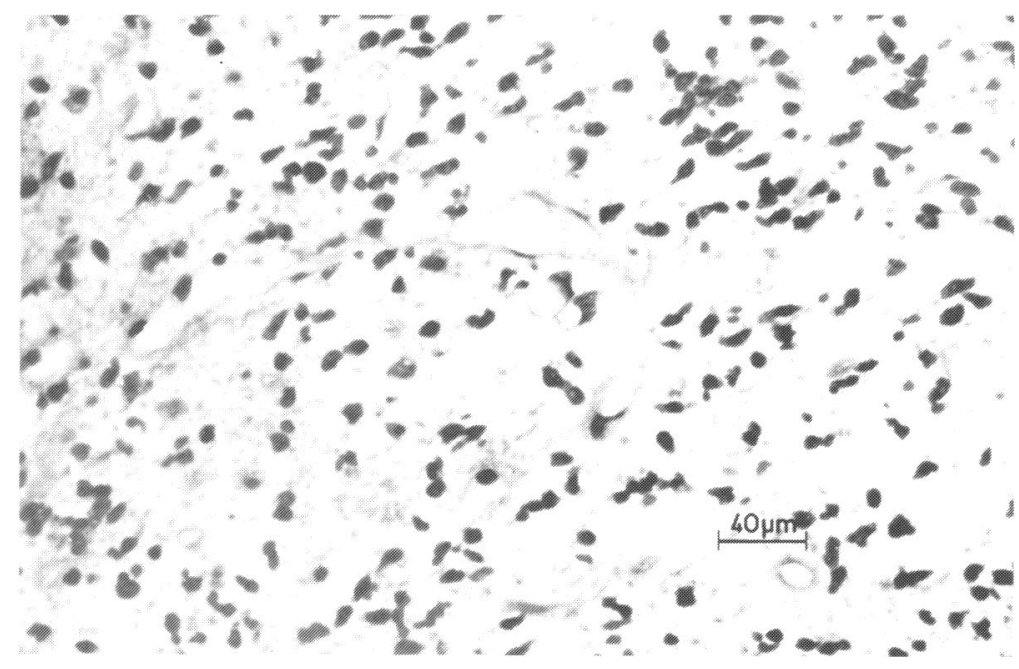

Fig. 3 Histopathological section from case 2, showing a myxoid liposarcoma with delicate vascular channels, lipoblasts, a few mitotic figures and some giant or pleomorphic cells. $H$ and $E$. 


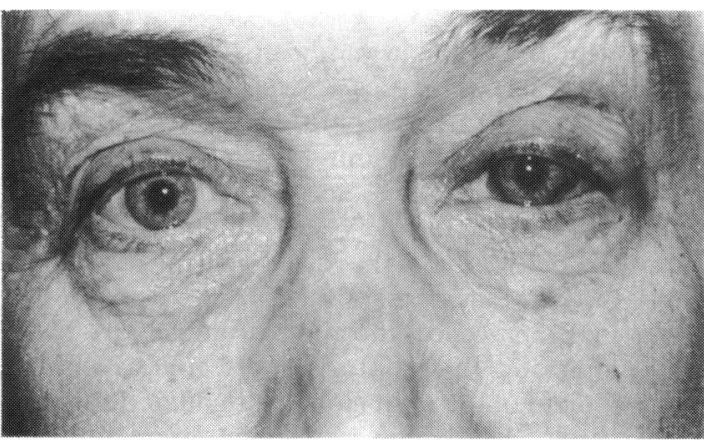

Fig. 4 Clinical picture of case 3, with swelling above the left lateral canthus, $1 \mathrm{~mm}$ of ptosis, conjunctival melanosis, and frontalis overaction.

asymptomatic when last seen in the clinic two years later.

\section{CASE 3}

A 77-year-old woman presented with lateral swelling of her right upper eyelid which had gradually increased over the previous nine months. Her general health was good.

On examination the visual acuities were $6 / 9$ in the right and $6 / 4$ in the left eye. A smooth, mobile, rubbery mass measuring $15 \mathrm{~mm}$ by $15 \mathrm{~mm}$ could be palpated in the superotemporal quadrant of the left orbit, but there was no displacement of the globe (Fig. 4). A millimetre of left blepharoptosis was associated with reduced function of the levator palpebrae superioris. Extensive conjunctival melanosis was present. Orbital $x$-rays were normal. An anterior trans-septal approach was used to biopsy a mass of gelatinous grey material involving the lacrimal gland.

Histological sections showed partial replacement of the lacrimal gland by stellate or spindle shaped cells and mast cells with a moderate degree of vascularity. There were multiple cytoplasmic vesicles characteristic of lipoblasts on electron microscopy (Fig. 5).

Computerised tomography failed to show residual tumour, but further gelatinous material was excised through an anterior approach. Complete removal would have required exenteration, but this was not considered justifiable because of the patient's age and slow tumour growth. Four months later she was stable with a left visual acuity of $6 / 9$ and a comfortable eye with a poor tear film. However, two years after the initial presentation there was increasing

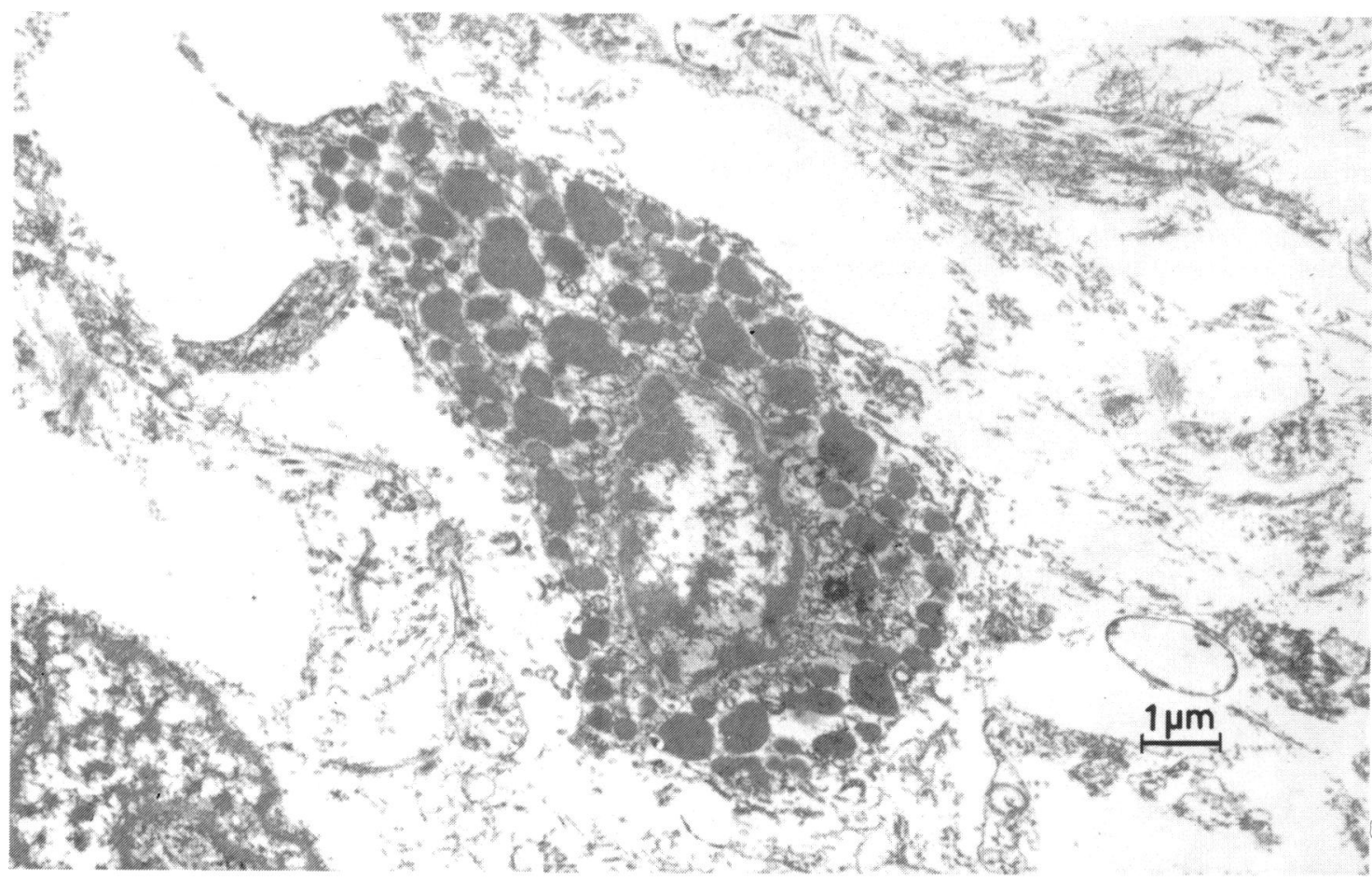

Fig. 5 Case 3: electron micrograph showing a cell replete with intracytoplasmic lipid surrounded by a loose collagenous stroma. 


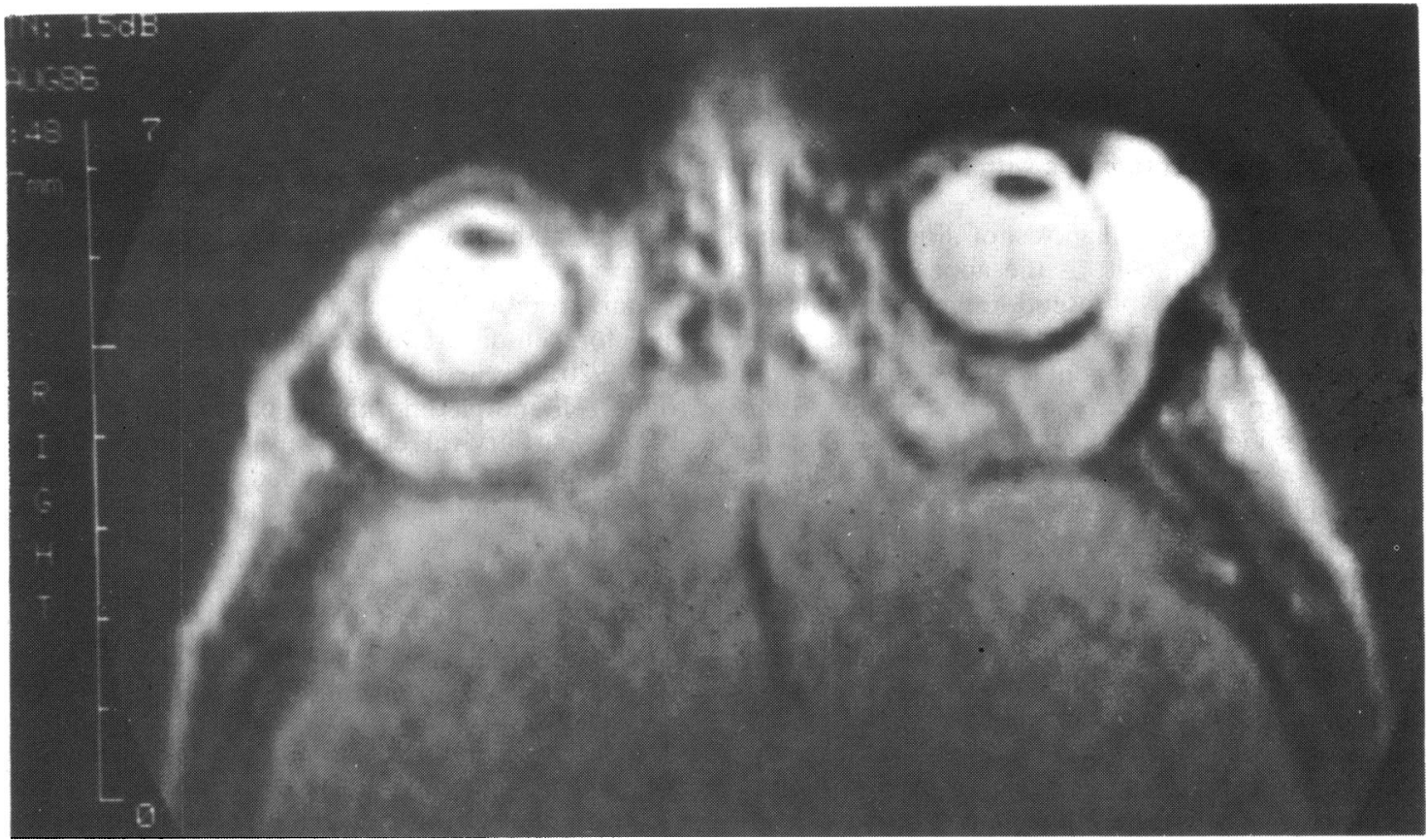

Fig. 6 Axial NMR scan of recurrent tumour in case 3, showing a high signal intensity on $T^{2}$ weighted spin echo sequences. The centre of the tumour is shown much more intensely than surrounding orbital fat.

discomfort with enlargement of the tumour. Nuclear magnetic resonance imaging revealed high signal intensity on $\mathrm{T}^{2}$ weighted spin echo sequences (Fig. 6). The spin characteristics were not those of a fat containing tumour. The centre of the lesion was clearly distinguishable, but the margins were difficult to distinguish. To prevent further spread of the tumour total exenteration of the left orbit was performed. Tumour extended to the equator inferiorly and was demonstrated in the edge of the histological sections. When the patient was last seen two months after surgery the socket was healing without evidence of further infiltration.

\section{Discussion}

Orbital liposarcoma is rare and difficult to diagnose at presentation because of inconsistent clinical features. A review of the literature revealed only 10 convincing reports of the tumour. Strauss ${ }^{2}$ described a case in the right orbit and Henderson ${ }^{3}$ described two, but neither author specified the type. Quere et $a l .{ }^{4}$ and Citolli $^{5}$ cited fatal primary orbital liposarcoma in childhood and Mortada reported it in a 37-year-old man. ${ }^{6}$ Only Citolli's case showed round cell preponderance. Stout's case ${ }^{7}$ and another reported by Schroeder et al. in $1978^{8}$ were myxoid. Jakobiec and Jones ${ }^{9}$ reported two cases in which young women were treated with surgery and 50 Gray of radiotherapy; the first received both treatments but died six years later of generalised metastases, and the second was treated for recurrence; she remained well at the time of the report. An anaplastic orbital secondary from a well-differentiated abdominal liposarcoma has recently been reported..$^{10}$ The patient was treated by orbital exenteration but died shortly after surgery.

The first case described here presented with rapid onset of unilateral proptosis that was initially attributed to thyroid eye disease. Infiltration of the medial rectus muscle was interpreted as muscle enlargement at the time of orbital decompression, and the surgery facilitated tumour spread through the disrupted periosteum into the maxillary and ethmoid sinuses. At a later stage computerised tomography revealed a large heterogenous mass with areas of negative attenuation compatible with fat. The other two smaller tumours were not well delineated on CT scans. Magnetic resonance imaging was not consistent with a high fat content in the third case. This is consistent with the gelatinous consistency of the tumour and remarkable paucity of lipid vacuoles on histological sections. In all three cases the diagnosis was established only when biopsy of tissue was performed.

At surgery liposarcoma gives a false impression of 
being well circumscribed or even encapsulated, with a distinct lobulated pattern. The myxoid type appears gelatinous, and orange colouring has been reported, but the three presented here were grey. Less differentiated forms are brain-like with haemorrhages and cysts.

Histopathological diagnosis of liposarcoma frequently proves difficult, as the appearance may be confused with that of pseudotumour, lipoma, or myxoma. Lipoblasts, which support the diagnosis, are not always prominent. Since Virchow ${ }^{11}$ first described myxoid liposarcoma 120 years ago, Stout ${ }^{7}$ and Enterline et al. ${ }^{12}$ have further classified the tumour, regarding it as one of the less malignant forms of liposarcoma.

The most recent Armed Forces Institute of Pathology (AFIP) classification by Enzinger and Weiss ${ }^{13}$ is based on the stage of lipoblast development, manifest by the proportions of cellular lipid and mucinous material in the extracellular spaces and the overall degree of cellularity and cellular pleomorphism. Enzinger and Weiss believe that the neoplasm takes its origin from primitive mesenchymal cells rather than mature adipose tissue. Myxoid liposarcoma, accounting for 40 to $50 \%$ of cases, comprises lipoblasts, including a characteristic 'signet ring' type, at all stages particularly at the tumour margin and a mucopolysaccharide matrix with a delicate capillary network. The paucity of mitotic figures may contrast with rapid growth. Round cell liposarcoma has fewer apparent blood vessels and myxoid tissue with aggressive cellular proliferation and a tendency to metastasise. Welldifferentiated liposarcoma usually resembles lipoma, though inflammatory cell infiltrates and fibrosis may occur. The dedifferentiated type occurs in only $5 \%$ of cases and metastasises in half of those, frequently producing both poorly and well differentiated deposits. Pleomorphic liposarcoma may feature eosinophilic giant cells similar to those in pleomorphic rhabdomyosarcoma or, less frequently, haphazardly arranged cells containing varying amounts of lipid.

In his series of liposarcoma of the lower limb and retroperitoneum Enterline et al. ${ }^{12}$ found the peak incidence to be between 40 and 60 years of age with a slight preponderance of males and higher proportion of myxoid tumours in young people. Enzinger and Winslow $^{14}$ noted an increased proportion of welldifferentiated and pleomorphic forms in older people. The 13 primary orbital liposarcomas described to date comprise seven males and six females ranging in age from 5 to 77 years, mean 35 years. Myxoid tumours were found in six, but the tumour type was not related to age. Local wide excision of liposarcoma occurring elsewhere in the body is advocated by most authors. ${ }^{1215-17}$ Enterline et al..$^{12}$ reported that myxoid tumours seemed to be the most radiosensitive whereas Spittle et al. ${ }^{16}$ was impressed by the response of poorly differentiated forms.

Kindblom et al. ${ }^{18}$ in a recent large series of systemic liposarcomas assessed the results of treatment in 77 patients; all had been treated by surgical excision of liposarcoma, and 27 had also received radiotherapy. A marked difference was found between the prognosis for well-differentiated and myxoid types (12/17 and 12/20 10-year survival respectively) and that for round cell and pleomorphic liposarcomas (1/13 and 0/21 10-year survival respectively). Tumour site, size, and completeness of excision had the greatest effect on prognosis, but radiotherapy did not significantly affect it.

Trials of chemotherapy have been discouraging. Spittle et al. ${ }^{16}$ reported one anecdotal case of pleomorphic liposarcoma with pulmonary metastases which responded to chemotherapy. Gottlieb et al. ${ }^{19}$ achieved complete remission in three out of seven cases of liposarcoma treated with both adriamycin and dimethyl triazenoimidazole carboxamide. Cytotoxic treatment is unlikely to influence myxoid liposarcoma because it has a slow rate of spread.

CONCLUSIONS

Orbital liposarcoma is a rare tumour which presents difficulties with clinical and histopathological diagnosis. The paucity of data on treatment for a neoplasm that is surprisingly aggressive leaves the clinician with a therapeutic dilemma. The management decision should be clarified by careful determination of the histological subtype, which is of the utmost importance in predicting the prognosis.

The aggressive pleomorphic and round cell types require radical surgery in all cases. In our opinion the commoner myxoid types necessitate exenteration or midfacial resection as a life-saving procedure in a young person. In elderly patients there may be a satisfactory, though probably temporary, response to local resection. Radical surgery should be performed if there is continued growth with increasing discomfort. Radiotherapy is considered to be relatively ineffective in the treatment of this tumour. ${ }^{18}$

We are grateful to Dr G A S Lloyd for providing and interpreting the CT scan in case 1 and to the Department of Medical Illustration for the clinical pictures.

\section{References}

1 Dahl E, Hammond HL, Sequiera E. Liposarcomas of the head and neck. J Oral Maxillofac Surg 1982; 40: 674-7.

2 Strauss M. Liposarckom der rechten Orbita. Dtsch Med Wochenschr 1911; 37: 239. 
3 Henderson JW. Orbital tumors. Saunders, 1973: Section II, ch. 9: 262-7.

4 Quere MA, Camain R, Baylet R. Liposarcoma orbitaire. Ann Oculist (Paris) 1963; 196: 994-1003.

5 Citolli P. Su di particolare forma di liposarcoma dell'orbita con cospicue variaziani morphologiche durante la sua evoluzione. Ann Ottalmol Clin Oculist 1964; 90: 326-37.

6 Mortada A. Rare primary orbital sarcomas. Am J Ophthalmol 1969; 68: 919-27.

7 Stout AP. Liposarcoma - the malignant tumor of lipoblasts. Ann Surg 1944; 119: 86-107.

8 Schroeder W, Dastendreck H, von Damarcus D. Primaeres myxoides Liposarkom der Orbit: klinischer und histopathologischer Fallbericht. Ophthalmologica 1976; 172: 337-45.

9 Jakobiec FA, Jones IS. In: Duane TD, ed. Clinical ophthalmology. Hagerstown: Harper and Row, 1982; 2: ch. 44.

10 Nasr AM, Ossoinig KC, Kerster RF, Blodi FC. Standardised echographic-histopathologic correlations in liposarcoma. Am J Ophthalmol 1985; 99: 193-200.

11 Virchow R. Myxoma lipomatodes malignum. Virchows Arch $1865 ; 32$ : 545-6.

12 Enterline HT, Culberson ID, Rochlin DB, Brady LW. Lipo- sarcoma: a clinical and pathological study of 53 cases. Cancer 1960; 13: 932-50.

13 Enzinger FM, Weiss SW. Liposarcoma. In: Soft tissue tumours. St Louis: Mosby, 1973; ch. 10: 242-80.

14 Enzinger FM, Winslow DJ. Liposarcoma: a study of 103 cases. Virchows Arch $(A)$ 1962; 335: 367-88.

15 Pack GT, Pierson JC. Liposarcoma: a study of 105 cases. Surgery 1954; 36: 687-712.

16 Spittle ME, Newton KA, McKenzie DH. Liposarcoma. A review of 60 cases. Br J Cancer 1970: 24: 696-704.

17 Kinne DW, Chu FCH, Hnvos AG, Yogoda A, Fortnes JG. Treatment of primary and recurrent retroperitoneal liposarcoma. Twenty-five years' experience at Memorial Hospital. Cancer 1973; 31: 53-6.

18 Kindblom LG, Angeevall L, Svendsen P. Liposarcoma: a clinicopathologic, radiographic and prognostic study. Acta Pathol Microbiol Scand (A) 1975: suppl 253.

19 Gottlieb JA, Baker LH, Quagliana JM, et al. Chemotherapy of sarcomas with a combination of adriamycin and dimethyl triazeno imidazole carboxamide. Cancer 1972; 30: 1632-8.

Accepted for publication 24 September 1987. 